\title{
Statistics of Large Diameter Radio SNRs.
}

\author{
Abdul I. Asvarov \\ Institute of Physics, 33 H. Javid Ave, 370143 BAKU, Azerbaijan
}

\begin{abstract}
It is shown that if the recently discovered hypernova events are intrinsically energetic spherically symmetric explosions of massive stars than they can leave after themselves very large diameter (up to 500 pc) nonthermal radio shells with spectral properties like ordinary shell type radio supernova remnants (SNRs). The properties of such shells are modeled and compared with the statistics of large diameter SNRs.
\end{abstract}

\section{Introduction}

The models for super energetic SN events presented to date tend to fall into two classes - an intrinsically energetic explosion of a massive progenitor star (hypernova) and more normal SNe artificially brightened by beaming. At least the spectra of several events (SNe 1998bw and 1997ef) are much better reproduced with the hypernova models than with the ordinary SN Ic model (Nomoto et al.,2000). If the first type models are realized, then such events should leave after themselves the remnants similar to usual SNRs but with sizes at least a few hundred pc. So, the investigation of very large diameter SNR can help to answer the question about the nature of objects known as hypernova.

The physics of the hypernova remnants (HNR) is expected to be the same as in the case of typical SNR and can be described by self similar solution until radiative cooling becomes important. In this paper we applied the model of radio emission of very extended old SNRs which is based on the assumptions: a) electrons are accelerated diffusively at the main shock front from thermal energies; test particle approximation is used; b) magnetic field in the shell is typical interstellar field compressed at the shock front; c)the influence of external pressure is included by using the approximation of Cox \& Anderson (1982) for the structure and kinematics of the remnant.

\section{Results}

The shape of the evolutionary tracks depends mainly on the electron density $n_{e}$ and the energy of SN explosion $E_{S N}$. An another input parameter of the model is the total pressure (thermal gas, $P_{t h},+$ magnetic field, $P_{m}$ ) of the ISM which determines the maximum diameter of SNR. This quantity is parametrized by $\beta=P_{t h} / P_{m}$ and the value of the magnetic field strength $H$. The input parameter which characterizes the mechanism of acceleration is the injection parameter $\phi$ (Bell, 1978). In all models we use $\beta=1$ and $\phi=410^{-3}$.

In Fig 1 the results of model calculations are represented in the empirical $\Sigma-D$ diagram. Our sample of SNRs consists of Galactic shell and composite 


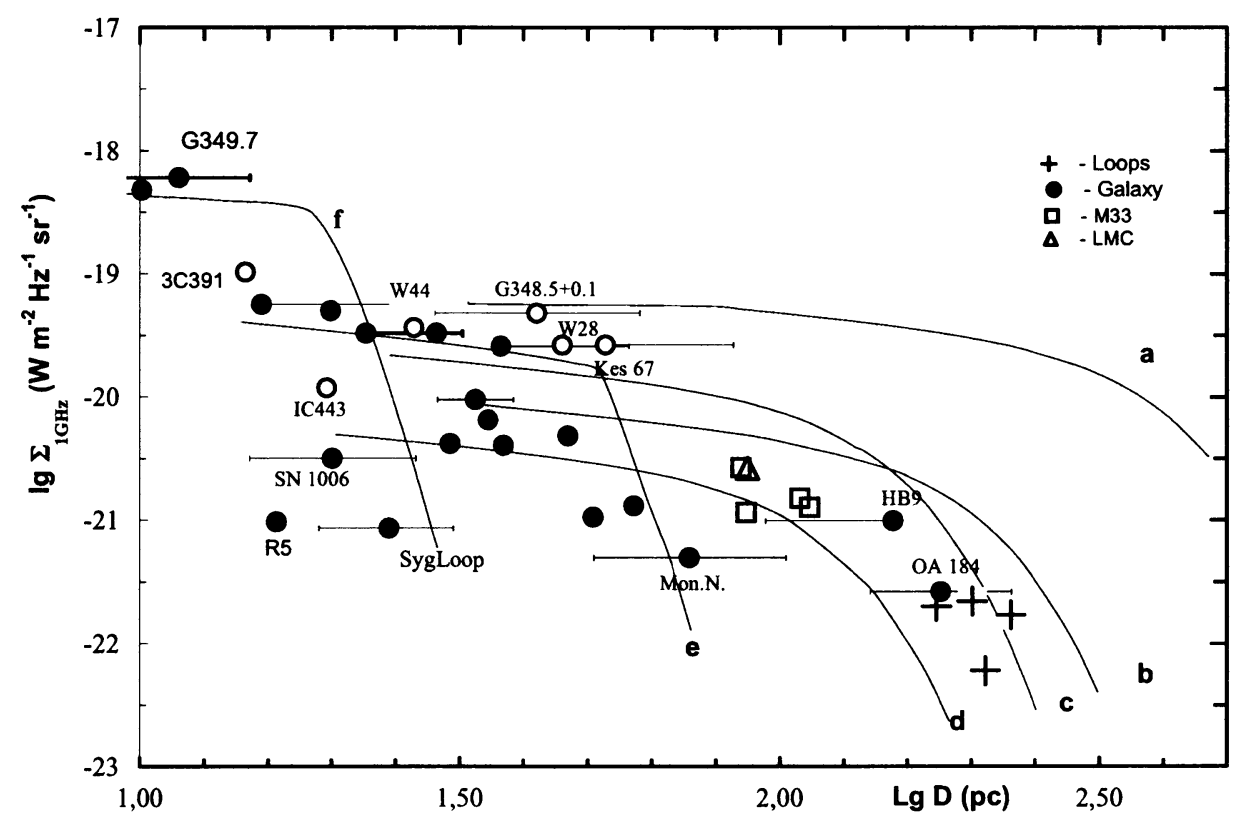

Figure 1. $\quad \Sigma-D$ diagram at $1 \mathrm{GHz}$ for the largest shell-like SNRs. Open circules are the composite type SNRs. Tracks are drown for: a) $n_{e}=510^{-2}, H_{0}=3 ; E_{S N}=100 ;$ b) $\left(5 \cdot 10^{-2} ; 3 ; 5\right)$; c) $\left(5 \cdot 10^{-2} ; 5\right.$; $5)$; d) $\left(5 \cdot 10^{-2} ; 3 ; 1\right)$; e) $(0.5 ; 5 ; 1)$; f) $(1 ; 5 ; 1) . n_{e}$ is in $\mathrm{cm}^{-3}, H_{0}$ is in $\mu G, E_{S N}$ is in $10^{51} \mathrm{erg}$.

type SNRs with $D>10 \mathrm{pc}$ from the catalogue of Green (1998) and 4 Galactic Loops from Berkhuisen (1986), 4 largest SNRs in M33 from(Duric et al. 1995), and SNR 0450-705 in LMC (Mathewson et al. 1985). Note that the largest known SNRs can be explained with $E_{S N} \leq 510^{51} \mathrm{erg}$. The existence of HNRs is problematical because it is difficult to detect such remnants in our Galaxy due to their low $\Sigma$ but they can be detected in nearby galaxies: at $10 \mathrm{Mpc}$ above modeled $\mathrm{HNR}$ can be detected as nonthermal source with $\alpha \simeq 0.6, S_{1 \mathrm{GHz}} \simeq 1$ mJy and $\theta \simeq 6^{\prime \prime}$.

\section{References}

Bell, A.R. 1978, MNRAS, 182, 443

Berkhuijsen, E.M. 1986, A\&A, 166, 257

Cox, D.P. \& Anderson, P.R. 1982,A.pJ, 253, 268

Duric, N., Gordon, S.M., Goss,W.M., et al.,1995,ApJ, 445, 173

Mathewson, D.S., Ford, V.L., Tuohy I.R. et al.1985, ApJS, 58, 197

Nomoto, K., Mazzali, P.A. \& Nakamura, T. 2000 astro-ph, 0003077 